\title{
Automated system for modeling traffic of multiservice networks
}

\author{
B.Ya. Likhttsinder ${ }^{1}$, A.V. Kharkovsky ${ }^{1}$, S.Yu. Antsinov ${ }^{1}$ \\ ${ }^{I}$ Povolzhsky State University of Telecommunications and Informatics, LevTolstoy street, 23, 443010, Samara, Russia
}

\begin{abstract}
The paper deals with the system for modeling parameters of traffic of multiservice networks, developed by the authors on the basis of Visual Studio, in C\#. The system is based on the principles of interval method of flow analysis general systems of mass service. The results of comparison with similar products are presented. Software structural scheme and its performance are analyzed with examples of video and Poisson traffic stream. The possibility of approximation coefficients determination is given which are coefficients of generalized KhinchinPollaczek formula. As a result, for real traffic, average size of the queues were determined by using approximation coefficients applications at different load factors, as well as a number of other characteristics of multiservice traffic, such as dispersion, correlation, probability distribution.
\end{abstract}

Key words: modeling; multi-service network; generalized Khinchin-Pollaczek formula; traffic analysis; packets

\section{Introduction}

The traffic of multiservice communication networks with packet switching is rather nonuniform [1], [2], [4]. The incoming packets are grouped at one time and virtually absent in others. The features of distribution function of the number of applications on the time intervals for the flow of multiservice networks were examined previously [7], [9]. It was shown that there are periods with different activity, which alternate time after time with different appearance probabilities, due to the fact that the number of claims in multiservice networks is often irregular. In each period there is only one flow. The lack of claims corresponds to the period with zero activity.

All the results given in [5] were obtained with the corresponding program, written in MatLab. The software, developed in the MatLab system, is well suited for scientific research; it is also suitable for analysis of traffic in real networks. Using the experience gained previously, the authors developed a system that allows you to quickly and accurately determine the main characteristics of traffic of multiservice communication networks, suitable for analysis of queues.

\section{Software structure}

The structural scheme of Fig. 1 shows the algorithm of the program.

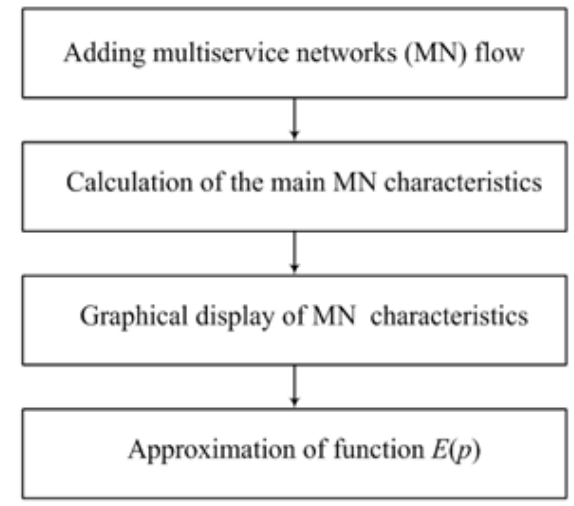

Fig.1. Software structure.

The first step is a user input file that contains information about the arrival of the packets meanwhile the operation of a multiservice network (obtained, for example, using the WireShark program [10]).

The second step is processing the file. The main traffic characteristics (time delays between packets, the expected number of packets in the queue at different load factors, etc.) are calculated. The next step is to build selected characteristics in the graphics area of the software window

At the last stage, approximating the numerator function of the generalized Khinchin-Pollyaczek formula, a user can determine coefficients that characterize this traffic, examined in [5].

\section{Analysis of software correctness}

The basis of this software lay down the methods of analysis and processing algorithms of traffic, given in [3], [8], [9]. To assess the correct operation of software, a comparison was made between previously defined video streaming characteristics and characteristics obtained from the analysis using the developed system. Below are the graphs obtained using developed system analysis. 


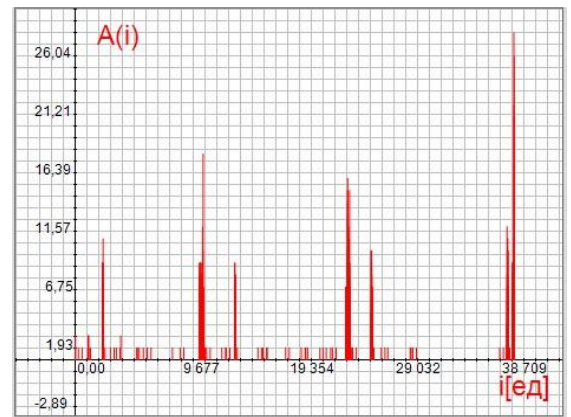

Fig.2. The number of packets at the intervals $\tau$ when the load factor $\rho=0.1$

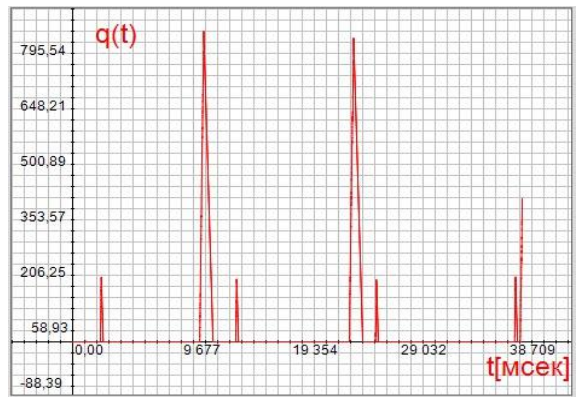

Fig.3. The number of packets in the queue at the intervals $\tau$ when the load factor $\rho=0.1$

The resulting graphs Fig. 2 and Fig. 3 are completely analogous to those examined in [6].

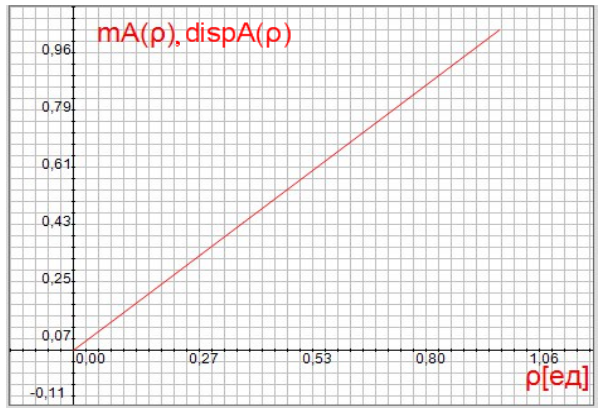

Fig.4. Dispersion and mathematical expectation of the number of packets on intervals $\tau$ for Poisson traffic stream.

The developed program was tested on Poisson traffic stream. In Fig. 4 demonstrated the graphs of dependencies of average value and dispersion of the packets at intervals corresponding to different load factors of the system $\rho$. Both graphs are linear and completely coincide, which is typical for Poisson traffic stream.

\section{Main features of the program}

Interface of the main program window consists of three blocks (Fig. 5):

1. Panel with flows.

2. Graph.

3. Setting bar of graph display.

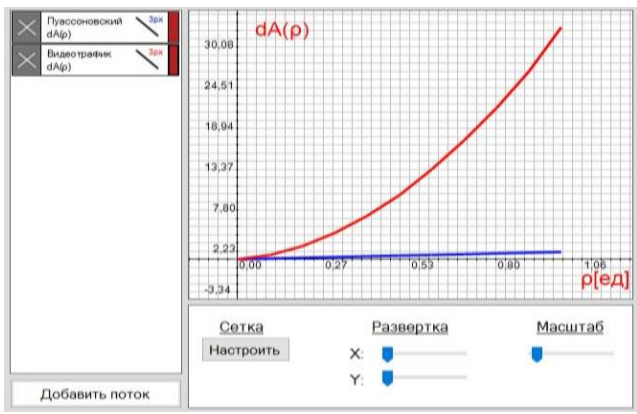

Fig. 5. The interface of the MSS traffic analysis program. 
Examine each block in more details. The system provides the addition to a flow, using the "Add flow" button in the user interface (Fig. 6).

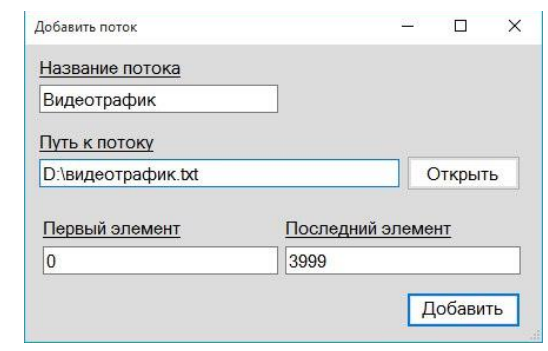

Fig.6.Interface adding flow.

The user has the ability to specify a name of the flow and limit the number of packets of input traffic. The flow file must contain information about the arrival times of packets or the intervals between packets.

The program is not limited to the number of flows and packets therein. You can add multiple flows and compare their characteristics, as shown in Fig. 5. After addition, the title of the flow is displayed on the panel (Fig. 7).

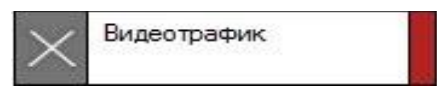

Fig.7. Graphical representation of flow.

The graphical representation of flow consists of three "buttons":

1. Remove the flow from the panel.

2. Display setting.

3. Shading the flow from the general graph.

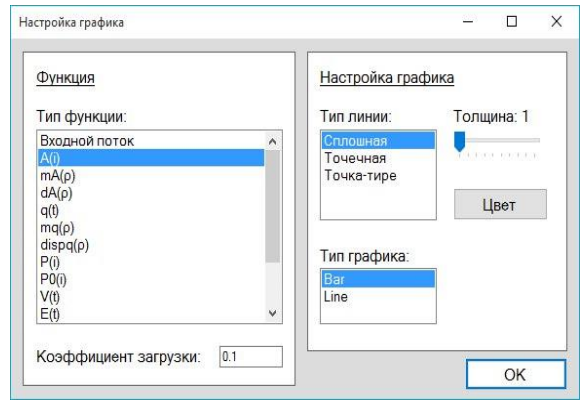

Fig. 8. Setting interface for flow display.

In the display settings dialog box of the graph (Fig. 8), a user can select traffic characteristic to build, tune in the graph (color, thickness and line type). When you click "OK", graphical representation of the flow changes, and selected characteristic is built.

The graph displayed in the Cartesian coordinate system is automatically scaled by the size of the window. The user has the ability to scale the graph, shift the plane graphics using the mouse, and reset these values and determine the coordinates of the point on the graph using the context menu (right mouse button). You can adjust the grid, sweep along the axes in the panel for setting the graph display.

\section{Determination of approximation coefficients}

The main practical benefit of this software is the ability to determine the coefficients of the approximation of the numerator of generalized Khinchin-Pollaczek formula [5], [6], [8]. It is necessary to build a characteristic of numerator dependence $m E(\rho)$ of Khinchin-Pollaczek formula on the load factor $\rho$ and to approximate it by a function $F(\rho)=\alpha\left(\rho-\rho_{0}\right)^{2}+\beta\left(\rho-\rho_{0}\right)$.

By changing the coefficients $\alpha, \beta$ and $\rho_{0}$, an approximation is made automatically, the results of which are shown in the diagrams Fig. 9.

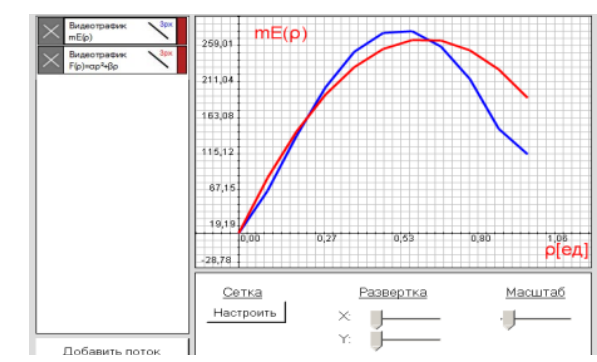

Fig.9. Approximation of characteristics $m E(\rho)$ of video stream. 
The obtained coefficients determine the generalized formula of Khinchin-Pollaczek:

$$
\overline{q(\rho)}=\frac{m E(\rho)}{2(1-\rho)}=\frac{\alpha\left(\rho-\rho_{0}\right)^{2}+\beta\left(\rho-\rho_{0}\right)}{2(1-\rho)} .
$$

Using the generalized Khinchin-Pollaczek formula, the average values of the queues are determined for different load factors. In Fig. 10 shows the real values of the queues and the values obtained as a result of approximation.

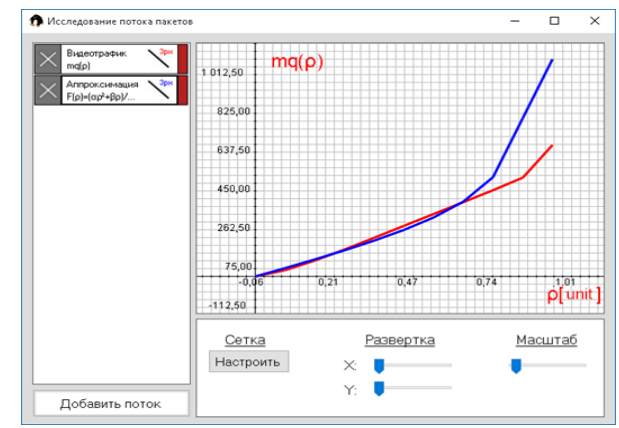

Fig.10. The numbers of packets in the queue, obtained using generalized Khinchin-Pollaczek formula and as a result of approximation.

\section{Conclusion}

The developed software can be used in the analysis of traffic of multiservice telecommunication networks. Further development involves automatic collection of information about the flow and processing in real time, allowing to obtain average values of coefficients of e generalized Khinchin-Pollaczek formula.

\section{References}

[1] Stepanov SN. Teletraffic theory. Concepts, models, applications. Moscow: Goryachaya liniya-Telecom Publ., 2015; 808 p. (in Russian)

[2] Kleinrock L. Communication Nets; Stochastic Message Flow and Delay. New York: McGraw-Hill, 1964.

[3] Sveshnikov AA. Applied methods of a random function theory. Moscow: Nauka Publ., 1968; 460 p.

[4] Martin Jh. A System analysis of data transmission. Englewood Cliffs, NJ: Prentice-Hall, 1972.

[5] Likhttsinder BY. Interval method of traffic analysis in multiservice communication systems. A supplement to journal Infokommunikacionnye tehnologii 2013; 8: 104-152. (in Russian)

[6] Likhttsinder BY. Interval analysis method of access networks. Samara: PSUTI Publ., 2015; 121 p. (in Russian)

[7] Likhttsinder BY. Correlation features of queue sizes in queueing systems with common type flows. Infokommunikacionnye tehnologii 2015; 13(3): 276280. (in Russian)

[8] Likhttsinder BY. About some generalizations of Pollaczek-Khinchin formula. Infokommunikacionnye tehnologii 2007; 5(4): 253-258. (in Russian)

[9] Likhttsinder BY. Correlation connections in batch flows of queuing systems. Telecommunications 2015; 9: 8-12.

[10] Sanders C. Practical Packet Analysis: Using Wireshark to Solve Real-World Network Problems. No Stach Press, 2017. 